\title{
Sistema de información para la gestión de los servicios de transporte utilizando la arquitectura REST en Lima-Perú
}

\section{Information system for the management of transportation services using REST architecture in Lima-Peru.}

\author{
Hillary Karin Bautista Vilchez ${ }^{1}$, Esteban Fernando Castillo Camizán², y Frank Edmundo Escobedo \\ Bailón ${ }^{3}$
}

\begin{abstract}
${ }^{1}$ Universidad Nacional Tecnológica de Lima Sur (UNTELS). Av. Cesar Vallejo S/N, sector 6 grupo 5, mz. P, sublote 19 - Villa El Salvador, Lima, Perú. Correo electrónico: hillarybautista2301@ gmail.com ${ }^{2}$ Universidad Nacional Tecnológica de Lima Sur (UNTELS). Av. Alfonso Ugarte S/N, mz. B2, sublote 9 - San Camilo, Lima, Perú. Correo electrónico: castilloesteban2009@gmail.com ${ }^{3}$ Universidad Nacional Tecnológica de Lima Sur (UNTELS). Calle Carlos Alayza y Roel 2392 Dpto. 104 Lince, Lima, Perú. Correo electrónico: fescobedo@ untels.edu.pe. ORCID: https://orcid.org/0000-0002-20580976
\end{abstract}

\section{Resumen}

Los servicios de transporte ayudan a las personas a trasladarse y a mover bienes de un lado a otro, ya sea por motivos comerciales o de cambio de vivienda. En el Perú, debido al COVID 19 el gobierno estableció medidas para aminorar las consecuencias del virus, lo que dejó a un grupo extenso de personas sin trabajo, afectando al sector transporte cuyo servicio se realiza en su mayoría de forma tradicional "no digitalizado o sistematizado", de esta forma los diferentes negocios o rubros fueron sufriendo una transformación tecnológica forzosa para poder continuar sobreviviendo en el mercado. El objetivo principal es analizar la viabilidad del desarrollo de un sistema de información haciendo uso de la arquitectura REST para los servicios de transporte en Lima - Perú, teniendo en cuenta que este se aplica principalmente al traslado de personas y bienes. Durante el desarrollo se analizó el mercado de los transportes de carga, la gestión de calidad del servicio de transporte, el cual tiene diferentes etapas además cada una de estas cuenta con sus propias dificultades tanto para el cliente como para quien realiza el servicio, por ello para el desarrollo del sistema de información se debe tener en cuenta el modelo de negocio, es decir cubrir una o más etapas, por otro lado se estudia la influencia de las tecnologías de información y comunicaciones (TICs) en este sector. Así mismo, se busca realizar una comparativa de las metodologías para el desarrollo del software

Palabras clave: servicios de transporte, arquitectura rest, plataformas digitales, transformación digital

\begin{abstract}
The transportation services help people to move and move goods from one place to another, either for commercial reasons or to move house. In Peru, due to COVID 19, the government established measures to lessen the consequences of the virus, which left a large group of people without work, which affected the transportation sector whose service is mostly performed in a traditional way "not digitized or systematized", thus the different businesses or items were undergoing a forced technological transformation in order to continue surviving in the market. The main objective is to analyze the feasibility of developing an information system using REST architecture.for transportation services in Lima - Peru, taking into account that it is mainly applied to the movement of people and goods. During the course, the freight forwarding market, the quality management of the transport service, which has different stages and each of these has its own difficulties for both the customer and for those who
\end{abstract}


perform the service, so for the development of the information system the business model must be taken into account, that is to cover one or more stages, on the other hand, the influence of information and communication technologies (TICs) in this sector is studied. Likewise, a comparison of the methodologies for software development is sought.

Keywords: transportation services, rest architecture, digital platforms, digital transformation.

\section{Introducción}

En el Perú, actualmente el servicio de transporte ha tenido un incremento debido a la pandemia COVID 19, muchas personas han optado por hacer uso de este servicio para mudarse a lugares aledaños a su trabajo por el motivo de no correr el riesgo de contagio, pérdida del empleo u otro. Además, la digitalización de otros sectores importantes para el mercado como las ventas por internet han impulsado el crecimiento de este proceso, ya que en la actualidad los productos que se comercializan pueden ser enviados a cualquier destino independientemente del tamaño o peso que lo caracteriza.

Las microempresas que son las que dan mayor trabajo en el Perú han tenido que realizar recortes, que en muchos casos han sido masivos tomando como punto crítico alguna dificultad o problema del trabajador. Esta problemática también originó que las personas trasladen sus lugares provisionales de vivienda a alquileres más bajos.

En el movimiento restringido dentro de suelo nacional, las empresas han restablecido su negocio paulatinamente, por lo que el traslado de cargas mostró un incremento debido a la compra de materiales y traslado de bienes.

Además, se toma en cuenta que según (Kee, 2018) en el diario El Peruano, la frecuencia de traslado por mudanzas, carga de bienes es significativamente alta en lo que respecta a los cálculos mensuales, debido a que este servicio es fundamental para la movilización de la producción a nivel nacional se recomienda que el proceso debe ser más eficiente, por esta razón el proceso de transporte de personas o bienes así como otros rubros están en la búsqueda de formas de digitalización, tomando como ejemplo Uber, Easy Taxi, Rappi, Glovo, entre otros.

En el presente trabajo se revisaron diferentes investigaciones con respecto a los servicios ofrecidos en el sector. En el año 2019, se propuso un diseño para una aplicación móvil de servicio de transporte de mudanzas en Colombia, la iniciativa comenzó debido a la exigencia de los clientes de la empresa de mudanzas E\&L Moving Services por el crecimiento tecnológico, en respuesta a ello la organización optó por la adaptación de su modelo de negocio a un sistema basado en aplicativos móviles, donde sus clientes puedan 
adquirir el servicio, dar seguimiento a los transportes de carga, así como poder dar su opinión acerca de la atención. (Mejía \& Parrado, 2019)

La adaptación del modelo de negocio resultó satisfactoria para la organización debido a que hubo una significativa ampliación en la segmentación de los clientes.

Un plan de gestión es importante para el correcto funcionamiento de los procesos dentro del sector transporte, en el plan de negocios para la creación de la aplicación móvil DESTWAY, para la gestión de viajes terrestres intermunicipales en Colombia, se recalca que el uso de aplicativos móviles aprovecha mejor las características de un teléfono inteligente que una plataforma web, por lo que se buscan adaptar los procesos de reserva, seguimiento, optimización de rutas a un dispositivo móvil. (Galvis \& Giraldo, 2015)

Por otro lado, el desarrollo de un aplicativo móvil de transporte entre la comunidad universitaria con capacidad de geolocalización para el proyecto Ud. sobre ruedas, se logró implementar un sistema basado en aplicativos móviles y la detección de ubicación. El proyecto parte de la respuesta positiva a la prueba piloto de viajes colectivos dirigido a los estudiantes de la comunidad universitaria para disminuir las dificultades de tráfico y seguridad a través de Facebook, se decidió crear un aplicativo móvil que se encargue de este proceso, de esta forma poder difundir el servicio a más estudiantes, así mismo recalca que la mayoría de los estudiantes hacen uso de los teléfonos inteligentes, debido a ello se busca aprovechar los beneficios nativos como geolocalización. El desarrollo se realiza con la estructura tecnológica Ionic, que permite la compilación para dispositivos Android e iOS, las funcionalidades se realizaron con la arquitectura Web Service Rest para el consumo de servicios. (Guzman \& Chaparro, 2017)

En Perú, se realizó una propuesta para el desarrollo del aplicativo TRANSTEC para el servicio de mudanza integral en la empresa mencionada. Se planteó la adaptación del modelo de negocio de la organización debido al crecimiento tecnológico y la demanda en su rubro, por lo cual se realizó un aplicativo móvil que permitiera cubrir la necesidad de la búsqueda de empresas que ofrecen este servicio. (Velasquez et al., 2017)

Desde otro punto de vista, se menciona que los aplicativos móviles en Perú han traído beneficios y desventajas para los consumidores, esto último se recalca debido a que el gobierno no tiene contemplado en sus leyes modelos de negocios virtuales de este rubro. Mediante un análisis a términos y condiciones, políticas de privacidad, así como la lectura a contrataciones en aplicativos como Rappi, Globo, entre otros. Se menciona la 
UNIVERSIDAD NACIONAL DE UCAYALI

Revista de Investigación Universitaria

Versión electrónica $2664-8423$

ARTICULO DE REVISIÓN
Vol. $11 \mathrm{~N}^{\circ} 1$, pp. 549 - 557, enero/junio 2021

Recibido 18/02/2021

Aceptado 11/06/2021

Publicado 30/06/2021 importancia de que las organizaciones que pasen por una transformación digital integral deben delimitar la responsabilidad de los usuarios del sistema tanto empresa, usuario final y agentes que intervienen durante el servicio, de esta forma poder ofrecer calidad.

(Vidal, 2019)

\section{Servicio de transporte}

Como menciona (Terrazos, 2021), el transporte es un componente importante dentro de la logística de cualquier empresa o en la vida cotidiana de las personas. La demanda de transporte de carga es el resultado de la interacción que se da entre los productores y compradores de bienes y servicios, ya que los productores tienen la necesidad de adquirir servicios de transporte para distribuir su producción hacia el cliente final satisfaciendo sus necesidades.

El sector transporte actualmente no está altamente desarrollado en el Perú. Si bien es cierto como indica (Vassallo, 2018), existen empresas como Cabify y Uber que han desarrollado nuevos modelos de negocios, como la economía colaborativa o bajo demanda, brindando servicios de intermediación entre conductores y clientes que solicitan el uso de servicio de transporte. Aún existe un gran porcentaje de este sector que operan bajo la informalidad y esto en muchos casos se debe a que las empresas o personas que brindan el servicio informal no cuentan con los recursos, medios o la capacitación necesaria para su evolución.

De acuerdo al diario (La República, 2019), el caos vehicular es el resultado de contar con servicios informales de transporte y la gran cantidad de vehículos antiguos que no deberían encontrarse en circulación. En el Perú, según estadísticas del año 2019 se contaba con un taxi por cada 50 habitantes, mientras que en Nueva York se contaba un taxi por cada 690 personas, lo cual refleja un problema grave en el sector que puede afectar en la sostenibilidad ambiental, la calidad del servicio brindado, etc.

\section{Gestión de calidad}

Según indica (Hernández et al., 2018), en la actualidad el diseño organizacional de las empresas adquiere cada vez mayor importancia en términos que se refieren a la calidad, eficacia y eficiencia de sus productos o servicios que brindan, para lo cual buscan optimizar recursos materiales, humanos, financieros, etc. La gestión de calidad es una estrategia, la cual asegura la competitividad empresarial, de esta manera permite entender a la organización como un conjunto de procesos interrelacionados cuyo objetivo está en lograr la satisfacción ante las necesidades más exigentes por parte de los clientes.

Según (Melendez, 2017), la gestión de calidad 
se basa en los procesos de retroalimentación, para lo cual se deben establecer un conjunto de políticas, objetivos y procesos que permitan mejorar el desarrollo de los productos o servicios de la organización, por ello es relevante escuchar adecuadamente a los clientes y poder evaluar sus percepciones a través de diferentes aplicaciones como el uso de encuestas, bajo un enfoque tradicional. Una actividad importante dentro de la gestión de calidad es el seguimiento de cuyos procesos generan errores, contratiempos y dificultades que en muchos casos genera desconfianza en los clientes. Por ellos es importante poder identificar dichas situaciones y actuar de forma acertada para mantener el prestigio, la posición y la imagen de la empresa.

\section{Tecnologías de la información y comunicaciones}

El objetivo principal de las TICs, según (Ayala \& Gonzales, 2015), es mejorar y dar soporte a los procesos de operación.

Debido a la innovación en tecnologías surgió un cambio significativo en el sector de transporte, por ese motivo las empresas como Cabify y Uber tomaron este desarrollo como una oportunidad de negocio con la generación de aplicaciones digitales que permite llegar tanto a conductores como a clientes. (Vassallo, 2018)
En los últimos años, la tecnología conocida como "la nube" ha tomado fuerza, debido a que facilita la obtención de datos en diferentes partes del mundo. Los servicios de transporte no son la excepción ya que esta información es de utilidad para recopilar el seguimiento de los suministros, bienes o carga, así mismo la inteligencia artificial que permite tomar la ruta óptima para el traslado, entre otras funcionalidades. Ello también ha repercutido en el análisis de logística, donde el internet de las cosas y el análisis predictivo está tomando un papel importante.

\section{Metodologías de desarrollo de software}

Según (Donoso, 2018), En el desarrollo de sistemas informáticos tenemos metodologías tradicionales y ágiles. La primera es conocida como desarrollo en cascada, las ventajas de esta es su cotidianidad, y además está orientada a resultados, por ello promueve el trabajo efectivo, siguiendo la premisa de primero definir, después diseñar y por último codificar, es por ello que surgen las siguientes desventajas en donde las personas muestran dificultades para seguir la linealidad, además que requiere demasiado tiempo para poder ver el producto y cualquier cambio implica una inversión elevada de tiempo, esfuerzo y dinero.

Por el contrario, una metodología ágil usa métodos de desarrollo iterativo e incremental de esta forma toma en cuenta la necesidad y 
dificultad del desarrollo. Algunas ventajas son:

la rápida respuesta a reformas durante el cambio de requerimientos, la voz del cliente durante en el proceso permitiendo la retroalimentación, entregas significativas y entre algunas de sus desventajas está la casi nula existencia de documentación, necesidad de un vínculo estrecho con los líderes y soluciones poco acertadas en etapas extensas.

Algunas metodologías tradicionales, según (Dallos et al., 2019), son las siguientes:

- $\quad$ El Rational Unified Process (RUP): tiene como objetivo el análisis a áreas o procesos grandes donde se plantean casos de uso y manejo de riesgos.

- Microsoft Solution Framework (MSF): cuyo objetivo es encontrar mejores prácticas al desarrollo centrándose en las especificaciones funcionales, enfocado en la resolución de errores.

- Administración de proyectos utilizando PMBOK: recomendado para proyectos de diversas áreas de conocimiento, pero para proyectos inestables en desarrollo puede ser más una dificultad.

Por otro lado, tenemos algunas metodologías ágiles, según (Dallos et al., 2019), son:

- SCRUM: tiene como objetivo disminuir riesgos, resultados en corto tiempo, estimaciones, retrospectiva de avances y fallas, marco para XP o RUP.

- Metodología Extreme Programming (XP): su objetivo es involucrar a todos los miembros de un mismo departamento, se desarrolla en base a las prioridades, estimaciones y entregables.

- Metodología Crystal Clear: cuyo objetivo es la comunicación de inicio a fin con el cliente, los avances de entregas son frecuentes.

En el contexto de ágiles frente a tradicionales, tenemos que la aplicación de alguna metodología va de acuerdo a lo que se requiere desarrollar, los objetivos planteados y la duración máxima del proyecto. Para tiempos cortos se recomienda metodologías ágiles, por sus entregables iterativos, en el caso de plazos largos, alcance integral, alto presupuesto $\mathrm{y}$ funcionalidades complejas se recomienda una metodología tradicional.

\section{Arquitectura Rest}

Rest se define como un estilo arquitectónico cuyos objetivos están basados en la escalabilidad de la interacción con los componentes, ya que en la actualidad existe una gran variedad de clientes (ordenadores y softwares) que pueden acceder a través de la web, estaciones de trabajo, aplicativos móviles, etc. gracias al uso de protocolos Hypertext Transfer Protocol (HTTP), como segundo objetivo permite la extensibilidad del sistema 
mediante el uso de las cabeceras que viajan a través de los Uniform Resource Identifier (URI)s, permitiendo la comunicación y el acceso a nuevos métodos y tipos de contenido. (Ruelas, 2017)

Algunas ventajas de la aplicación de esta arquitectura en un negocio, según (Ribas, 2018), las cuales son:

- Reducen los costes de mantenimiento e incrementa la agilidad de los procesos de transformación de las empresas, lo cual mejora la calidad y la funcionalidad del sistema y por lo tanto el rendimiento de los procesos dentro de la organización,

- Separa la lógica entre el cliente y el servidor, mejorando la portabilidad y el desarrollo de la interfaz en diferentes tecnologías, Además aumenta la escalabilidad de los proyectos y permite que los distintos componentes que se desarrollen puedan evolucionar de forma independiente.

- Visibilidad, fiabilidad y escalabilidad ya que es evidente que la separación entre cliente y servidor permite que el sistema pueda escalar sin riesgo.

\section{Conclusiones}

La presente investigación da a conocer que la implementación de un sistema de información dirigido a la gestión de transporte permite mejorar la calidad del servicio que las empresas puedan brindar a sus clientes, transformando los procesos en búsqueda de su mayor eficiencia, así como identificar recursos ociosos y distribuir eficazmente los recursos necesarios. Además, estos tipos de sistemas permiten conocer la situación actual de las empresas, que da a conocer y evaluar aquellas carencias o falencias presentes que no permiten su crecimiento, de tal manera que los directivos puedan evaluar estrategias en base a retrospectivas realizadas internamente como organización involucrando el compromiso con los objetivos de la empresa y la mejora continua de la misma, así como también externas considerando las necesidades y expectativas de los clientes que frecuentemente se actualizan.

De la inspección de los casos de éxitos una gran parte de los resultados indica que los sistemas orientados al rubro de transporte se centran en ciertas problemáticas de este sector como son los procesos de: planificación, búsqueda, seguimiento, entre otros. Además, se debe considerar en el desarrollo los planteamientos que correspondan a la delimitación de responsabilidades de los usuarios finales del sistema.

La presente investigación confirma la viabilidad del desarrollo de un sistema para el sector de transporte que cubra las necesidades o problemáticas de este sector, así mismo se verifica la oportunidad de mercado en los 
UNIVERSIDAD NACIONAL DE UCAYALI

Revista de Investigación Universitaria

Versión electrónica $2664-8423$

ARTICULO DE REVISIÓN
Vol. $11 \mathrm{~N}^{\circ} 1$, pp. 549 - 557, enero/junio 2021

Recibido 18/02/2021

Aceptado 11/06/2021

Publicado 30/06/2021 servicios de transportes de carga en Perú. De igual manera un sistema de información basado en la arquitectura REST es la más recomendable para el desarrollo de aplicativos móviles debido a que permite portabilidad, escalamiento y desarrollo de interfaces en diferentes tecnologías.

Con un sistema basado en la Arquitectura REST mediante el uso de plataformas móviles y web, se puede realizar una transformación digital a nivel de flujo de negocio para una organización, esto ayuda a ofrecer una asistencia de calidad brindando seguimiento del servicio, facilidades para adquisición, soporte al cliente, entre otros.

La metodología recomendada a usar para proyectos de plazo corto y enfocada a entregables son las metodologías ágiles, ya que sigue el lineamiento de iteraciones que dan un producto significativo relacionado al cliente final, por tanto, una metodología ágil permite tener una relación cercana entre desarrolladores y la MYPE, supliendo ajustes o cambios a tiempo.

Finalmente se concluye que la metodología ágil recomendada para el desarrollo del sistema es SCRUM, debido a que está orientada a entregables y mantiene una continua mejora en las retrospectivas, además se recomienda tener como punto crítico la documentación de los flujos de negocio del sistema para la escalabilidad del proyecto.

\section{Referencias bibliográficas}

Ayala, E. E., \& Gonzales, S. R. (2015). Tecnologías de la Información y la Comunicación [Universidad Inca Garcilaso de la Vega]. http://repositorio.uigv.edu.pe/bitstrea $\mathrm{m} / \mathrm{handle} / 20.500 .11818 / 1189 /$ Libro TIC \%282\%29-1-76 $\% 281 \% 29$.pdf?sequence $=1 \&$ is Allowe $\mathrm{d}=\mathrm{y}$

Dallos, L. P., Ariza, D. C., Moncada, D. S., \& Franco, V. J. (2019). Análisis comparativo entre metodologías ágiles y tradicionales para la gerencia de proyectos [EAN Universidad]. En Journal of Chemical Information and Modeling (Vol. 01, Número 01). https://repository.ean.edu.co/bitstream /handle/10882/9559/MoncadaDaniel2 019.pdf? sequence $=1 \&$ is Allowed $=\mathrm{y}$

Donoso, E. (2018, agosto 18). Desarrollo de Aplicaciones Tradicionales y Ágiles Servicios Agiles. https://serviciosagiles.com/desarrolloaplicacion

Galvis, J. A., \& Giraldo, J. A. (2015). Plan de negocios para la creación de la aplicación móvil destway, para la gestión de viajes terrestres intermunicipales [Universidad Tecnológica de Pereira]. https://core.ac.uk/download/pdf/71398 379.pdf

Guzman, N. R., \& Chaparro, M. (2017). Desarrollo de aplicación movil de transporte entre la comunidad universitaria con capacidad de geolocalizacion para el proyecto ud sobre ruedas [universidad Distrital Francisco José de Caldas]. http://repository.udistrital.edu.co/bitstr eam/handle/1 1349/6267/ChaparroAriz aMiguelLeonardo2017.pdf;jsessionid= 2F3C6921DE106B063EDA73E9D26 68BAA? sequence $=1$ 
UNIVERSIDAD NACIONAL DE UCAYALI

Revista de Investigación Universitaria

Versión electrónica $2664-8423$

ARTICULO DE REVISIÓN
Vol. $11 \mathrm{~N}^{\circ} 1$, pp. 549 - 557, enero/junio 2021

Recibido 18/02/2021

Aceptado 11/06/2021

Publicado 30/06/2021
Hernández, H. G., Barrios, I., \& Martínez, D. (2018). Gestión de la calidad: elemento clave para el desarrollo de las organizaciones. Criterio Libre, 16(28), 179-195.

https://core.ac.uk/download/pdf/229911 313.pdf

Kee, F. Mac. (2018, junio 27). Herramienta Para El Transporte Terrestre Tecnología online. $\quad \mathrm{El}$ peruano. https://elperuano.pe/suplementosflippin g/economika/270/web/pagina03.html

La República. (2019, junio 22). Hay más de 68 mil taxis informales solo en Lima. https://larepublica.pe/economia/2019/06 /22/hay-mas-de-68-mil-taxisinformales-solo-en-lima/?ref=lre

Mejía, E., \& Parrado, L. E. (2019). diseño de una aplicación móvil de servicio de transporte de mudanzas en Colombia. http://repository.unipiloto.edu.co/bitstre am/handle/20.500.12277/6304/TRABA JO_DE_GRADO.pdf?sequence $=1 \&$ isAl lowed $=\mathrm{y}$

Melendez, A. (2017). Propuesta de implementación del sistema de gestión de calidad en una industria pesquera según la norma ISO 9001:2015 [Pontificia Universidad Católica Del Perú]. En Pontificia Universidad Católica del Perú. http://tesis.pucp.edu.pe/repositorio/hand le/123456789/9337

Ribas, E. (2018). Qué es Api Rest y por qué debes de integrarla en tu negocio. Thinking for Innovation. https://www.iebschool.com/blog/quees-api-rest-integrar-negocio-businesstech/
Ruelas, D. A. (2017). Modelo de composición de microservicios para la implementación de una aplicación web de comercio electrónico utilizando kubernetes [Universidad Nacional Del Altiplano]. En Universidad Nacional del Altiplano.

http://repositorio.unap.edu.pe/handle/U NAP/6781

Terrazos, L. M. (2021). Propuesta de mejora en los procesos de una pequeña empresa del sector transporte terrestre de carga pesada.

https://repositorioacademico.upc.edu.pe /bitstream/handle/10757/625896/Terraz osA_L.pdf?sequence $=3 \&$ is Allowed $=y$

Vassallo, J. M. (2018). Nuevas tecnologías en la gestión de la movilidad. La revista de los Ingenieros de Caminos, Canales y Puertos, 84. http://ropdigital.ciccp.es/pdf/publico/20 18/2018_diciembre_3604.pdf

Velasquez, Y. M., Chávez, M. Y., León, M., \& Saldivar, M. (2017). Transtec SAC Servicio de Mudanza Integral [Universidad Peruana De Ciencias Aplicadas].

https://repositorioacademico.upc.edu.pe /bitstream/handle/10757/622989/CHAV EZ_RM.pdf?sequence $=5$ \&isAllowed $=\mathrm{y}$

Vidal, M. de L. (2019). Análisis de la responsabilidad de los servicios de delivery de comida por aplicativos móviles, en la ciudad de Lima Metropolitana, en el año 2019 [Universidad Peruana De Ciencias Aplicadas].

https://repositorioacademico.upc.edu.pe /handle/10757/631316 